\title{
ANALYSIS OF RAINFALL INTENSITY OF KUNIGAL TALUK, TUMKUR DISTRICT, KARNATAKA USING GIS TECHNIQUES
}

\author{
Nandeesha $^{1}$, Ravindranath. ${ }^{2}$, Shanthibhushan. $\mathbf{S}^{3}$ \\ ${ }^{1}$ Professor, Civil Engineering Department, Siddaganga Institute of Technology, Karnataka, India \\ ${ }^{2}$ Research Scholar, Civil Engineering Department, Siddaganga Institute of Technology, Karnataka, India \\ ${ }^{3}$ Student, Civil Engineering Department, Siddaganga Institute of Technology, Karnataka, India
}

\begin{abstract}
Land, water and soil are limited natural resources and their wide utilization with increasing population is a major area of concern. to mitigate the demand and supply gap between resources and ever increasing demand, it is of prime importance to conserve the natural resources with proper prioritization for its sustainable development. The present work mainly analysis of rainfall intensity for Kunigal Taluk. The study area of Kunigal taluk is located in southern part of Tumkur district in Karnataka state. The taluk covers an area of 981 Sq.km. and average rainfall of $802 \mathrm{~mm}$. The area is bounded by the latitude North $12^{\circ} 44^{\prime} 38.74^{\prime \prime}$ to $13^{\circ} 8^{\prime} 1.16^{\prime \prime}$ and the longitude East 76 49' 43"to 77 9' 57'. The main part of the area is covered under Survey of India (SOI) Toposheet numbers 57 C/16, 57 G/4, 57 D/13, 57 C/12, 57 D/9, and 57 H/1 (Scale 1:50,000) and having eight rain gauge stations each rain gauge stations of monthly of pre monsoon, south west monsoon and north east monsoon rainfall data is analyzed from 1901-2011. The study of uneven distribution of rainfall causes scarcity fresh water/potable water, agriculture purpose, etc. The water table fluctuation under the influence of rainfall and drought. The rain fall data is analyzed by time series and its components, and by conventional methods. Here we are mainly discussing about rainfall data and its intensity throughout the year, and water table fluctuation data by graphical method, and using software for the analysis of the rainfall to know variation of its value across a vast area in a systematic manner using Arc view $3.2 a$ software and DBF files.
\end{abstract}

Keywords: Rain gauge station, time series, water level fluctuation, Arc view 3.2 a etc...

\section{INTRODUCTION}

Rainfall is the key climatic variable that governs the regional hydrological cycle and engineering design projects including water design etc. Rainfall is the only source for both surface and ground water resources in the world. The evaporated water when condensed at the high altitude in the form of clouds, at high altitude due to reduction in the atmospheric pressure these water vapors expand by absorbing energy from the surrounding air, which cools down. The capacity of the atmosphere at the high altitude depends on its temperature, humidity, wind direction and wind speed. When it falls below the due point, it cannot retain the excessive moisture, which starts falling in the form of rain, hails, dew sleet, precipitation. Changing precipitation pattern, and its impact on surface water resources is an important climatic problem facing society today associated with global warming, there is strong indication that rainfall changes are already taking place on both the global and regional scales. Variation in the monsoon rainfall has both social and political impact in India, agricultural activities are largely depends on rain.

\subsection{Location of Study Area}

The study area of Kunigal taluk is located in southern part of Tumkur district in Karnataka state. The taluk covers an area of
981 Sq.km. and average rainfall of $802 \mathrm{~mm}$. The area is bounded by the latitude North $12^{0} 44^{\prime} 38.74^{\prime \prime}$ to $13^{0} 8^{\prime} 1.16^{\prime \prime}$ and the longitude East $76^{\circ} 49^{\prime} 43^{\prime \prime}$ to $77^{\circ}$ 9' $57^{\prime}$ '. The main part of the area is covered under Survey of India (SOI) Toposheet numbers $57 \mathrm{C} / 16,57 \mathrm{G} / 4,57 \mathrm{D} / 13,57 \mathrm{C} / 12,57 \mathrm{D} / 9$, and 57 H/1 (Scale 1:50,000). Tumkur district was formed in 1966 under Nandidurga division; the district is having geographical area of $10648 \mathrm{sq} . \mathrm{km}$. Tumkur district falls in the southern dry agro-climatic zone. The average temperature of district is $40^{\circ} \mathrm{C}$ .the location of study area is shown in Map No 1.

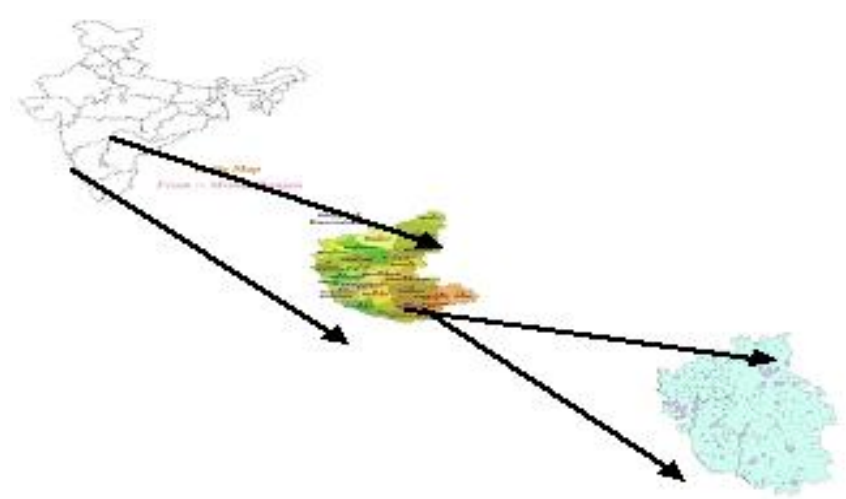

Map No 1: Location map of study area. 


\subsection{Measurement of Rainfall}

Rainfall and other forms of precipitation are measured in terms of depth, the values being expressed in millimeters. One millimeter of precipitation represents the quantity of water needed to cover the land with a $1 \mathrm{~mm}$ layer of water, taking into account that nothing is lost through drainage, evaporation or absorption. Instrument used to collect and measure the precipitation is called rain gauge. Rainfall varies greatly both in time and space with respect to time - Temporal variation, with space - Spatial variation. The temporal variation may be defined as hourly, daily, monthly, seasonal variations and annual variation (long-term variation of precipitation), and spatial variation is due to space, time and geographical area.

\subsection{Objectives of Study}

In the study area total 108 years rainfall data is collected and it is analyzed by the conventional methods and non conventional methods To analyze the rainfall data (1901 to 2011) of Kunigal taluk, Tumkur by a standard methods which is mentioned below. Interpretation of South-west, North-east monsoons over an area about 108 years.

\section{METHODOLOGY}

The rainfall details collected in monthly wise of 108 years in the eight locations in the Kunigal taluk prior existing rain gauge stations in taluk currently maintained by KSDMC and analyzed conventional methods such as arithmetic mean method Thiessen polygon method, and non conventional methods are Thiessen polygon using Arc GIS software. . Rain fall data is analyzed by time series followed by the trend variation, the seasonal variation, and also by moving average method of three years and five years.

\subsection{Computing Average Rainfall:-}

\subsubsection{Airthematic Mean Method:-}

$$
\bar{p}=\frac{p_{1}+p_{2} \ldots+p_{i}+\cdots+p_{n}}{N}=\frac{1}{N} \sum_{i=1}^{N} p_{i}
$$

\subsubsection{Thiessen Polygon Method}

$$
\bar{p}=\frac{\left\{P_{1} A_{1}+P_{2} A_{2} \cdots \cdots \cdots+P_{m} A_{m}\right\}}{\left(A_{1}+A_{2}+\cdots+A_{m}\right)}=\sum_{i=1}^{m} P_{i} \frac{A_{i}}{A}
$$

Where: The ratio $\left(\frac{A_{i}}{A}\right)$ is called the weight age factor of station $\mathrm{i}$.

\subsubsection{Moving Average Method}

$$
\mathrm{F}_{(\mathrm{t}+1)}=\frac{\sum(\text { Most Recent K data values })}{\mathrm{K}}
$$

i.e.,

$$
F_{(t+1)}=\frac{\left(A_{1}+A_{2}+A_{3}\right)}{K}
$$

For 3-years moving average similarly for 5- years where $F_{(t+1)}$ - Forecasting period values, t- Time / period A - Actual value of the time series in period.

\subsubsection{Drought Calculations:-}

$$
\% \text { drought }=\left(\frac{P(1,2,3 \ldots)}{\sum P}-1\right) * 100
$$

\section{RESULTS}

The table 1 shows the average annual rainfall from the 2001 to 2010 the maximum rainfall accrued the taluk during 2001-2010 is $883.94 \mathrm{~mm}$ and minimum rainfall is $668.77 \mathrm{~mm}$. Thiessen polygon area of the concern raingauge station is as shown below and the total entire area of the taluk will be 981 sqkm .

Table 1 Annual rainfall and Theissen polygon area

\begin{tabular}{|l|l|r|r|}
\hline SL.NO & & $\begin{array}{l}\text { Thiessen } \\
\text { ANNUAL } \\
\text { RAINFALL } \\
\text { polygon } \\
\text { area } \\
\text { Sqkm }\end{array}$ \\
\hline 1 & Amruturu & 786.97 & 131.6596 \\
\hline 2 & Bilidevalaya & 668.77 & 186.2881 \\
\hline 3 & Huliyurudugra & 877.24 & 216.9549 \\
\hline 4 & Kunigal S F & 855.48 & 82.24542 \\
\hline 5 & Kunigal T B & 798.09 & 54.47195 \\
\hline 6 & Markonahalli & 685.84 & 109.2605 \\
\hline 7 & Nidasale & 762.66 & 59.55078 \\
\hline 8 & Santhepete & 883.94 & 141.1155 \\
\hline 9 & Maximum & 883.94 & 216.9549 \\
\hline 10 & Minimum & 668.77 & 54.47195 \\
\hline
\end{tabular}

\subsection{Drought Calculations}

The table 2 is shown the drought calculations of the study area from the 1901 to 2010 of pre monsoon south west monsoon and north east monsoon the NR $=$ Normal rainfall, SLD $=$ slight drought, $\mathrm{MD}=$ Moderate drought, $\mathrm{SR}=$ Sever drought. 
Table 2 Drought calculations.

\begin{tabular}{|l|r|r|r|l|l|l|l|l|r|}
\hline & $\begin{array}{l}\text { total } \\
\text { rainfa } \\
\text { ll }\end{array}$ & $\begin{array}{l}\mathrm{N} \\
\mathrm{R}\end{array}$ & $\begin{array}{l}\mathrm{S} \\
\mathrm{L}\end{array}$ & $\begin{array}{l}\mathrm{M} \\
\mathrm{D}\end{array}$ & $\begin{array}{l}\mathrm{S} \\
\mathrm{D}\end{array}$ & $\begin{array}{l}\text { M } \\
\text { in } \\
\text {. }\end{array}$ & $\begin{array}{l}\text { Ma } \\
\text { x. }\end{array}$ & $\begin{array}{l}\text { Me } \\
\text { an }\end{array}$ & SD \\
\hline $\begin{array}{l}\text { Pre } \\
\text { monso } \\
\text { on } \\
(1-4)\end{array}$ & & & & & & & & & \\
\hline $\begin{array}{l}\text { south } \\
\text { west } \\
\text { monso }\end{array}$ & & 5 & 1 & 2 & & 5 & & & 73.1 \\
on & 413.0 & 6 & 1 & 2 & & 2 & & & 153. \\
$(5-8)$ & 3 & 0 & 8 & 3 & 8 & 3 & 864 & 398 & 1 \\
\hline north & & & & & & & & & \\
east & & & & & & & & & \\
monso \\
on (9-
\end{tabular}

Rainfall calculations of 108 years of pre monsoon, south west monsoon and north east monsoon as shown in table $3,4, \& 5$.

Table 3 pre monsoon rainfall statists

\begin{tabular}{|c|c|c|c|c|c|c|}
\hline $\begin{array}{c}\text { Year (1901- } \\
\text { 2011) }\end{array}$ & Jan & Feb & Mar & Apr & May & $\begin{array}{c}\text { Pre- } \\
\text { Monso } \\
\text { on }\end{array}$ \\
\hline AVG & $\begin{array}{c}2.2 \\
8\end{array}$ & $\begin{array}{c}2.8 \\
2\end{array}$ & $\begin{array}{c}11.4 \\
1\end{array}$ & 43.3 & 108.4 & 168.28 \\
\hline MIN & 0 & 0 & 0 & 0 & 4 & 54 \\
\hline MAX & 64 & 33 & 164 & 174 & 290 & 375 \\
\hline SD & $\begin{array}{c}7.9 \\
8\end{array}$ & $\begin{array}{c}6.9 \\
4\end{array}$ & $\begin{array}{c}30.7 \\
9\end{array}$ & 39.93 & 53.89 & 73.19 \\
\hline VAR & $\begin{array}{c}53 . \\
8\end{array}$ & $\begin{array}{c}43 . \\
5\end{array}$ & $\begin{array}{c}906 . \\
4\end{array}$ & $\begin{array}{c}1535 . \\
8\end{array}$ & $\begin{array}{c}2814 \\
1\end{array}$ & 5354.5 \\
\hline
\end{tabular}

Table 4 South west monsoon statists

\begin{tabular}{|c|c|c|c|c|c|}
\hline $\begin{array}{c}\text { Year (1901- } \\
\text { 2011) }\end{array}$ & Jun & Jul & Aug & Sep & $\begin{array}{c}\text { South } \\
\text { West } \\
\text { Monsoon }\end{array}$ \\
\hline AVG & 67.3 & 81.2 & 114.5 & 149.9 & 413 \\
\hline MIN & 0 & 11 & 4 & 0 & 123 \\
\hline MAX & 185 & 282 & 413 & 388 & 864 \\
\hline SD & 43.1 & 54.5 & 81.97 & 91.95 & 153.1 \\
\hline VAR & 1888.4 & 2976.7 & 6720.5 & 8456.5 & 23462.1 \\
\hline
\end{tabular}

Table 5 North East monsoon statists

\begin{tabular}{|c|c|c|c|c|c|}
\hline $\begin{array}{c}\text { Year (1901- } \\
\text { 2011) }\end{array}$ & Oct & Nov & Dec & $\begin{array}{c}\text { North } \\
\text { Monst } \\
\text { noo }\end{array}$ & Total \\
\hline AVG & 156.4 & 54 & 10.1 & 220.67 & 802 \\
\hline MIN & 0 & 0 & 0 & 10 & 292 \\
\hline MAX & 421 & 365 & 126 & 604 & 1322 \\
\hline SD & 91.55 & 57.3 & 18.9 & 111.22 & $\begin{array}{c}207.0 \\
8\end{array}$ \\
\hline VAR & $\begin{array}{c}8383 . \\
2\end{array}$ & $\begin{array}{c}3290 . \\
6\end{array}$ & $\begin{array}{c}359.0 \\
3\end{array}$ & 12370.5 & 42886 \\
\hline
\end{tabular}

\subsection{Thiessen Polygon Area}

The Thiessen polygon is studies performed by using Arc GIS 3.2a and Arc view software, fig 1 shows the construction of Thiessen polygon with concern rain gauge stations existing in Kunigal taluk, area of the each polygon is calculated and tabulated in table no 1 .

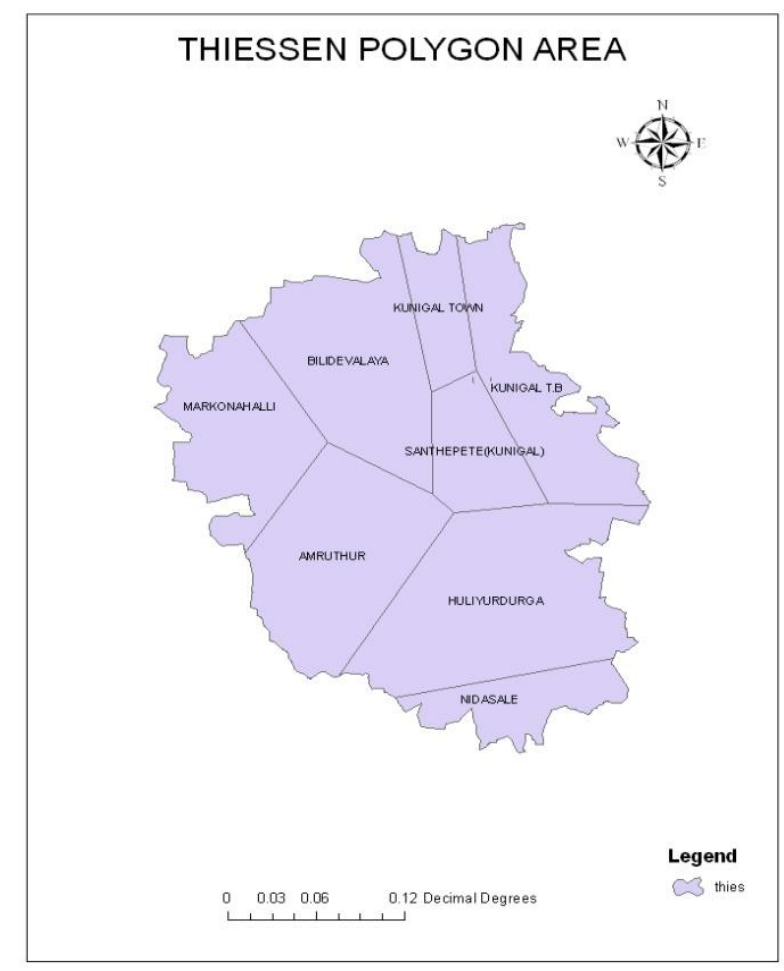

Fig 1 Thiessen polygons of Kunigal area 


\section{CONCLUSIONS}

The graph of pre monsoon, south-west \& north-east monsoons shows the increasing trend rainfall in which it shows Kunigal taluk receives normal rainfall of $802 \mathrm{~mm}$ annually, $413 \mathrm{~mm}$ in south-west Monsoon, $221 \mathrm{~mm}$ during North-East Monsoon and 168 during Pre-Monsoon season. Therefore, the average annual rainfall of Kunigal taluk is $802 \mathrm{~mm}$ and the standard deviation of this taluk is 20.708. Hence, the co-efficient of variation is 25.821 . From the three years and five years moving average calculations the rainfall for three and five years maximum and minimum rainfall will be notified and the average rainfall of the entire taluk from 1901-2011will be analyzed and trend analysis is tabulated. And the coefficient of variance $\mathrm{C}_{\mathrm{v}}$ of rainfall is about $25.82 \%$ during annually, $37 \%$ in South-West Monsoon, 50.4\% in North-East Monsoon \& $43.48 \%$ in PreMonsoon season. Maximum rainfall received during the end of September \& in the October and particularly during 40th to 45th standard Weeks. The trend line shows the increasing pattern of rainfall.

\section{ACKNOWLEDGEMENTS}

The special thanks to Dr V.S Prakesh Director and Dr Srinivasa reddy scientist KSDMC for the providing the rainfall data

\section{REFERENCES}

[1]. A.S Chandrabose et al. Development of fully distributed rainfall-runofff modal (FDRRM) using RS and GIS, Journal of applied hydrology vol XXIV No.3\&4,Jul\&Sep,-2011,pp76-83

[2]. C.R Suribabu et al. Evaluation of design rainfall depth from 100 years of daily rainfall -A case study, Journal of applied hydrology vol XXIV No.3\&4,Jul\&Sep,-2011,pp18-28.

[3]. Dr.NM Tipparudrappa et al Stastical analysis of rainfall distribution and trend rainall anomalies in the two districts of Karnataka , HYDRO 2010 pp362-369

[4]. M R Yadupathi putty et al. Rainfall variation over the last two decades a comparision between two districts of karanataka ISH Journal vol 13 no.2 sept 2007 pp 93-107.

[5]. N.R Patel et al. Probability Distribution Analysis of comsective Days Rainfall Data for Sabarkantha District of North Gujrath Region, India HYDRO 2007 pp 86-93.

[6]. Subramanya K, Engineering Hydrology.

[7]. www.aboutcivil.org/net/rainfall analysis

[8]. www.ksndmc.net

[9]. www.imd.com/weather at web

[10]. www.cgwb.in/bangalore.

\section{BIOGRAPHIES}

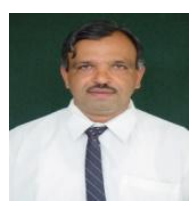

Professor, Civil Engineering Department, Siddaganga Institute of Technology, Karnataka, India

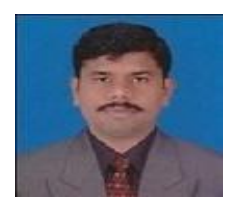

Research scholar, Siddaganga Institute of Technology, Civil Engineering Dept Tumkur

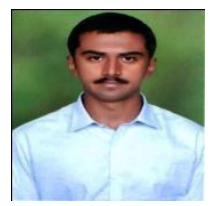

Student , SIT Civil Engineering department Tumkur 
The below table $(\mathrm{Q} 1=$ jan+feb+mar+apr) followed remaining Q2, Q3 \&Q4, shows the moving average of rainfall throughout the year in every monthly data is tabulated and formulated as show in table no 6.

Table 6 Rainfall data with seasonal

\begin{tabular}{|c|c|c|c|c|c|c|c|}
\hline YEAR & Q1 & Q2 & Q3 & Q4 & $\begin{array}{l}\text { Total } \\
\text { Rainfall }\end{array}$ & $\begin{array}{l}3 y r- \\
\text { moving } \\
\text { avg }\end{array}$ & $\begin{array}{c}\text { 5yr- } \\
\text { moving } \\
\text { avg }\end{array}$ \\
\hline 1901 & 16 & 220 & 468 & 307 & 1011 & - & - \\
\hline 1902 & 0 & 121 & 176 & 213 & 510 & - & - \\
\hline 1903 & 0 & 293 & 530 & 385 & 1208 & - & - \\
\hline 1904 & 0 & 158 & 258 & 175 & 591 & 909.67 & - \\
\hline 1905 & 41 & 158 & 236 & 117 & 552 & 769.67 & - \\
\hline 1906 & 11 & 128 & 766 & 241 & 1146 & 783.67 & 774.4 \\
\hline 1907 & 18 & 267 & 205 & 68 & 558 & 763 & 801.4 \\
\hline 1908 & 0 & 166 & 110 & 16 & 292 & 752 & 811 \\
\hline 1909 & 23 & 200 & 417 & 279 & 919 & 665.33 & 627.8 \\
\hline 1910 & 0 & 171 & 458 & 341 & 970 & 589.67 & 693.4 \\
\hline 1911 & 0 & 193 & 132 & 206 & 531 & 727 & 777 \\
\hline 1912 & 0 & 179 & 443 & 221 & 843 & 806.67 & 654 \\
\hline 1913 & 6 & 217 & 457 & 87 & 767 & 781.33 & 711 \\
\hline 1914 & 0 & 137 & 241 & 228 & 606 & 713.67 & 806 \\
\hline 1916 & 0 & 225 & 520 & 284 & 1029 & 738.67 & 743.4 \\
\hline 1917 & 70 & 269 & 545 & 175 & 1059 & 800.67 & 755.2 \\
\hline 1918 & 16 & 173 & 310 & 178 & 677 & 898 & 860.8 \\
\hline 1919 & 18 & 247 & 194 & 270 & 729 & 921.67 & 827.6 \\
\hline 1920 & 1 & 147 & 196 & 60 & 404 & 821.67 & 820 \\
\hline
\end{tabular}




\begin{tabular}{|c|c|c|c|c|c|c|c|c|}
\hline 1921 & 7 & 175 & 362 & & & 796 & 603.33 & 779.6 \\
\hline 1922 & 11 & 286 & 242 & & & 940 & 643 & 733 \\
\hline 1923 & 47 & 194 & 263 & & & 528 & 713.33 & 709.2 \\
\hline 1924 & 1 & 151 & 367 & & & 551 & 754.67 & 679.4 \\
\hline 1925 & 2 & 226 & 450 & & & 895 & 673 & 643.8 \\
\hline 1926 & 11 & 152 & 338 & & & 621 & 658 & 742 \\
\hline 1927 & 0 & 138 & 410 & & & 593 & 689 & 707 \\
\hline 1928 & 12 & 96 & 316 & & & 743 & 703 & 637.6 \\
\hline 1929 & 7 & 207 & 327 & & & 819 & 652.33 & 680.6 \\
\hline 1930 & 0 & 207 & 168 & & & 733 & 718.33 & 734.2 \\
\hline 1931 & 11 & 219 & 235 & & & 645 & 765 & 701.8 \\
\hline 1932 & 0 & 209 & 288 & & & 991 & 732.33 & 706.6 \\
\hline 1933 & 0 & 208 & 694 & & & 1097 & 789.67 & 786.2 \\
\hline 1934 & 0 & 140 & 107 & & & 452 & 911 & 857 \\
\hline 1935 & 0 & 172 & 463 & & & 937 & 846.67 & 783.6 \\
\hline 1936 & 82 & 190 & 404 & & & 851 & 828.67 & 824.4 \\
\hline YEAR & $\mathrm{Q}$ & 1 & 22 & Q3 & Q4 & $\begin{array}{l}\text { Total } \\
\text { Rainfall }\end{array}$ & $\begin{array}{l}\text { yr- } \\
\text { moving } \\
\text { avg }\end{array}$ & $\begin{array}{c}5 \mathrm{yr}- \\
\text { moving } \\
\text { avg }\end{array}$ \\
\hline 1937 & 2 & 8 & 89 & 191 & 159 & 667 & 746.67 & 865.6 \\
\hline 1938 & 1 & 3 & 31 & 625 & 10 & 779 & 818.33 & 800.8 \\
\hline 1939 & 4 & $\underline{2}$ & 64 & 287 & 604 & 1159 & 765.67 & 737.2 \\
\hline 1940 & c & 3 & 45 & 232 & 321 & 898 & 868.33 & 878.6 \\
\hline 1941 & c & 2 & 13 & 232 & 190 & 635 & 945.33 & 870.8 \\
\hline 1942 & c & 2 & 85 & 222 & 254 & 761 & 897.33 & 827.6 \\
\hline
\end{tabular}




\begin{tabular}{|c|c|c|c|c|c|c|c|}
\hline 1943 & 17 & 268 & 318 & 407 & 1010 & 764.67 & 846.4 \\
\hline 1944 & 142 & 245 & 336 & 176 & 899 & 802 & 892.6 \\
\hline 1945 & 0 & 298 & 222 & 95 & 615 & 890 & 840.6 \\
\hline 1946 & 18 & 123 & 309 & 330 & 780 & 841.33 & 784 \\
\hline 1947 & 9 & 144 & 241 & 115 & 509 & 764.67 & 813 \\
\hline 1948 & 7 & 241 & 222 & 157 & 627 & 634.67 & 762.6 \\
\hline 1949 & 0 & 83 & 417 & 296 & 796 & 638.67 & 686 \\
\hline 1950 & 0 & 170 & 332 & 276 & 778 & 644 & 665.4 \\
\hline 1951 & 0 & 305 & 382 & 143 & 830 & 733.67 & 698 \\
\hline 1952 & 0 & 217 & 297 & 416 & 930 & 801.33 & 708 \\
\hline 1953 & 0 & 216 & 230 & 351 & 797 & 846 & 792.2 \\
\hline 1954 & 5 & 341 & 247 & 171 & 764 & 852.33 & 826.2 \\
\hline 1955 & 39 & 309 & 449 & 222 & 1019 & 830.33 & 819.8 \\
\hline 1956 & 0 & 214 & 92 & 488 & 794 & 860 & 868 \\
\hline 1957 & 19 & 325 & 212 & 308 & 864 & 859 & 860.8 \\
\hline 1958 & 21 & 446 & 207 & 178 & 852 & 892.33 & 847.6 \\
\hline 1959 & 0 & 279 & 440 & 91 & 810 & 836.67 & 858.6 \\
\hline 1960 & 8 & 269 & 512 & 266 & 1055 & 842 & 867.8 \\
\hline 1961 & 10 & 246 & 109 & 198 & 563 & 905.67 & 875 \\
\hline 1962 & 22 & 270 & 339 & 248 & 879 & 809.33 & 828.8 \\
\hline 1963 & 0 & 155 & 202 & 286 & 643 & 832.33 & 831.8 \\
\hline 1964 & 0 & 182 & 496 & 361 & 1039 & 695 & 790 \\
\hline 1965 & 0 & 166 & 328 & 68 & 562 & 853.67 & 835.8 \\
\hline
\end{tabular}




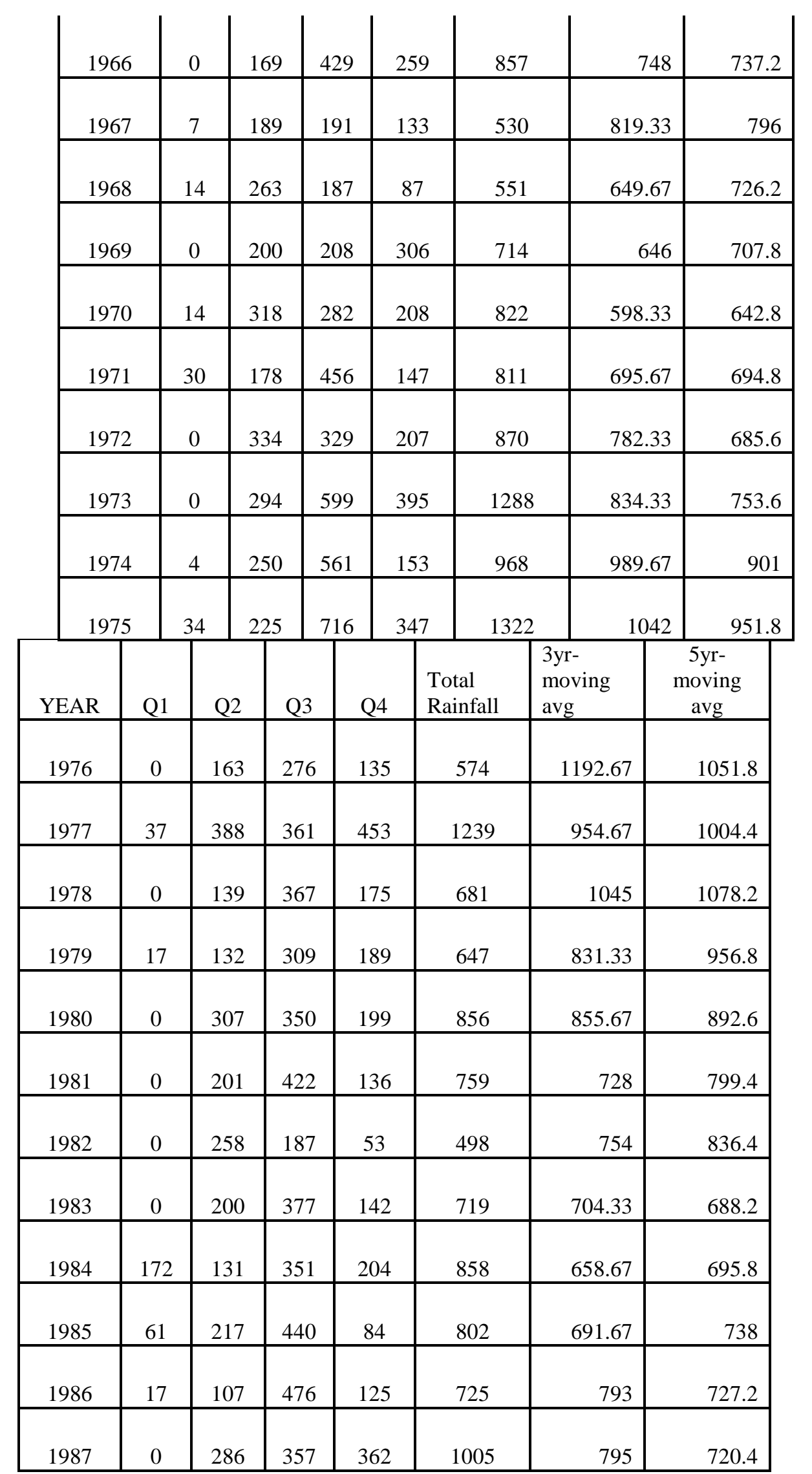




\begin{tabular}{|c|c|c|c|c|c|c|c|}
\hline 1988 & 36 & 274 & 838 & 86 & 1234 & 844 & 821.8 \\
\hline 1989 & 7 & 141 & 318 & 177 & 643 & 988 & 924.8 \\
\hline 1990 & 1 & 213 & 143 & 248 & 605 & 960.67 & 881.8 \\
\hline 1991 & 26 & 409 & 341 & 272 & 1048 & 827.33 & 842.4 \\
\hline 1992 & 0 & 294 & 370 & 243 & 907 & 765.33 & 907 \\
\hline 1993 & 0 & 277 & 389 & 243 & 909 & 853.33 & 887.4 \\
\hline 1994 & 64 & 158 & 210 & 208 & 640 & 954.67 & 822.4 \\
\hline 1995 & 18 & 121 & 388 & 108 & 635 & 818.67 & 821.8 \\
\hline 1996 & 6 & 235 & 521 & 146 & 908 & 728 & 827.8 \\
\hline 1997 & 0 & 227 & 462 & 169 & 858 & 727.67 & 799.8 \\
\hline 1998 & 0 & 123 & 602 & 183 & 908 & 800.33 & 790 \\
\hline 1999 & 4 & 199 & 401 & 390 & 994 & 891.33 & 789.8 \\
\hline 2000 & 6 & 143 & 372 & 345 & 866 & 920 & 860.6 \\
\hline 2001 & 0 & 153 & 370 & 207 & 730 & 922.67 & 906.8 \\
\hline 2002 & 29 & 173 & 146 & 187 & 535 & 863.33 & 871.2 \\
\hline 2003 & 35 & 66 & 201 & 236 & 538 & 710.33 & 806.6 \\
\hline 2004 & 5 & 427 & 504 & 126 & 1062 & 601 & 732.6 \\
\hline 2005 & 33 & 323 & 429 & 319 & 1104 & 711.67 & 746.2 \\
\hline 2006 & 134 & 178 & 43 & 144 & 499 & 901.33 & 793.8 \\
\hline 2007 & 0 & 337 & 479 & 144 & 960 & 888.33 & 747.6 \\
\hline 2008 & 162 & 228 & 436 & 185 & 1011 & 854.33 & 832.6 \\
\hline 2010 & 30 & 256 & 373 & 292 & 951 & 823.33 & 927.2 \\
\hline 2011 & 15 & 299 & 291 & 264 & 869 & 974 & 905 \\
\hline
\end{tabular}


The trend analysis map shows the slightly increasing trend but the rainfall is same because it is unevenly distributed. Shown in fig 2

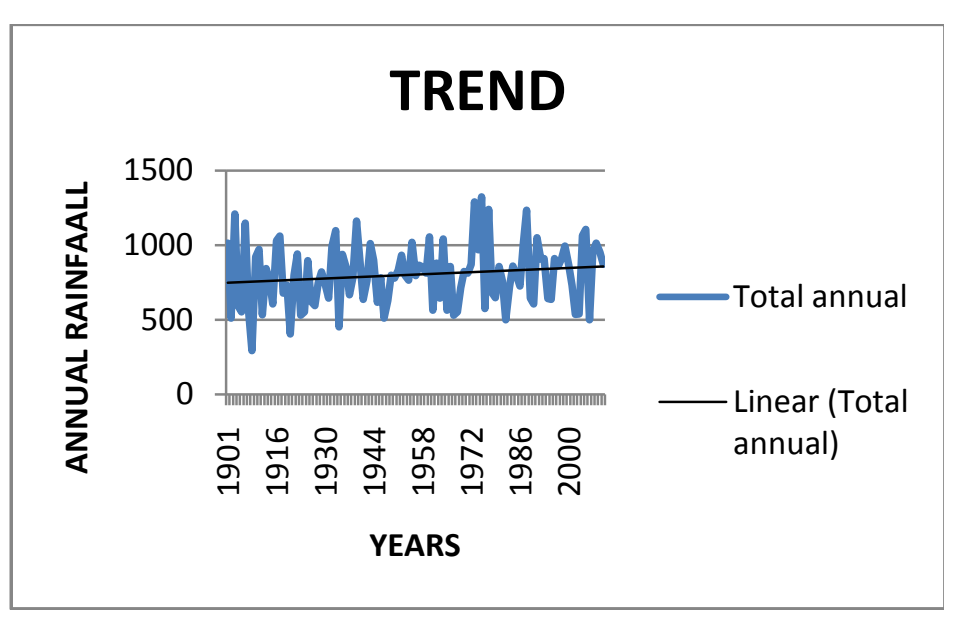

Fig 2 shows the trend analysis of rainfall (1901-2011)

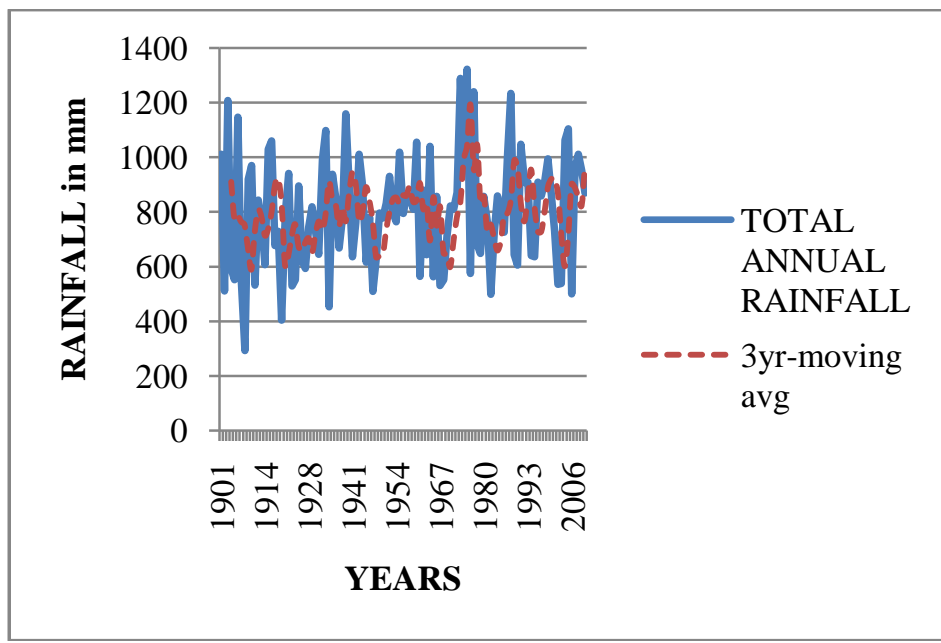

Fig 3 shows the 3y moving average of rainfall

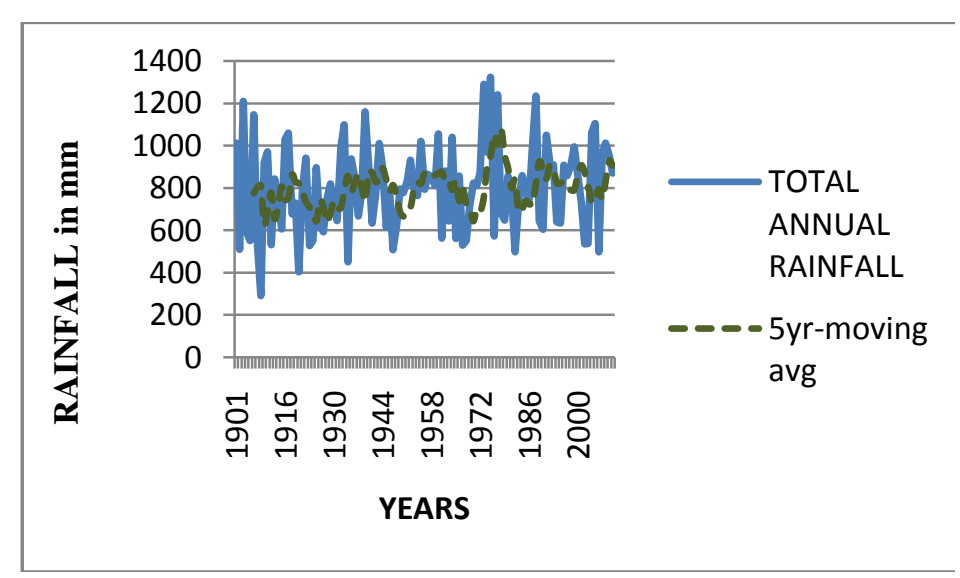

Fig 4 shows the 5y moving average of rainfall
Seasonal variation of rainfall (2001-2010) corresponding to table no 1 .is shown in fig 6.

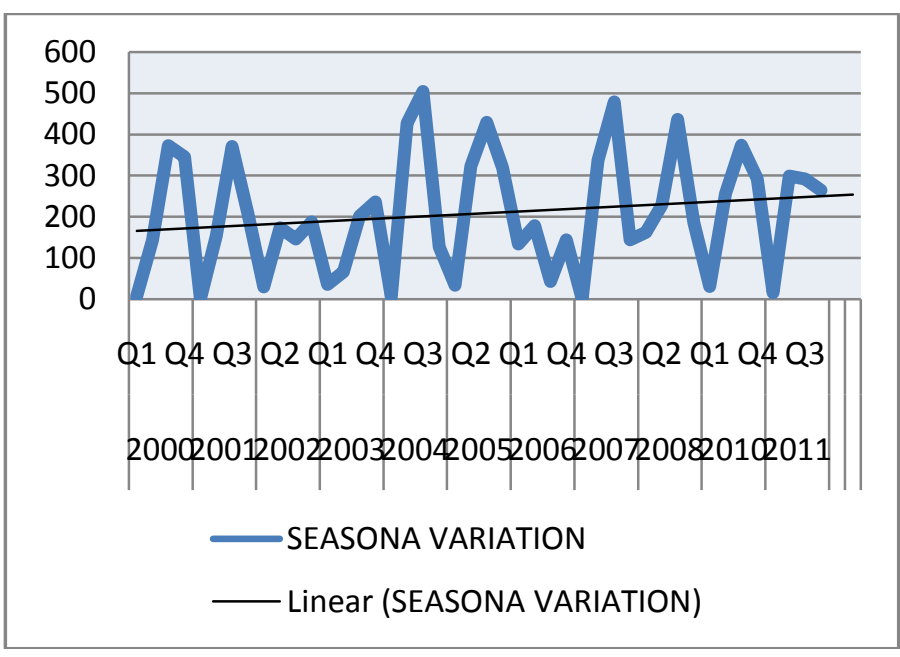

Fig 6 seasonal variation of rainfall

Seasonal variation of rainfall from 1901-2011 and each season wise like pre monsoon, southwest monsoon and North east monsoon is shown in the fig $8,9, \& 10$

\section{Seasonal variation of rainfall}

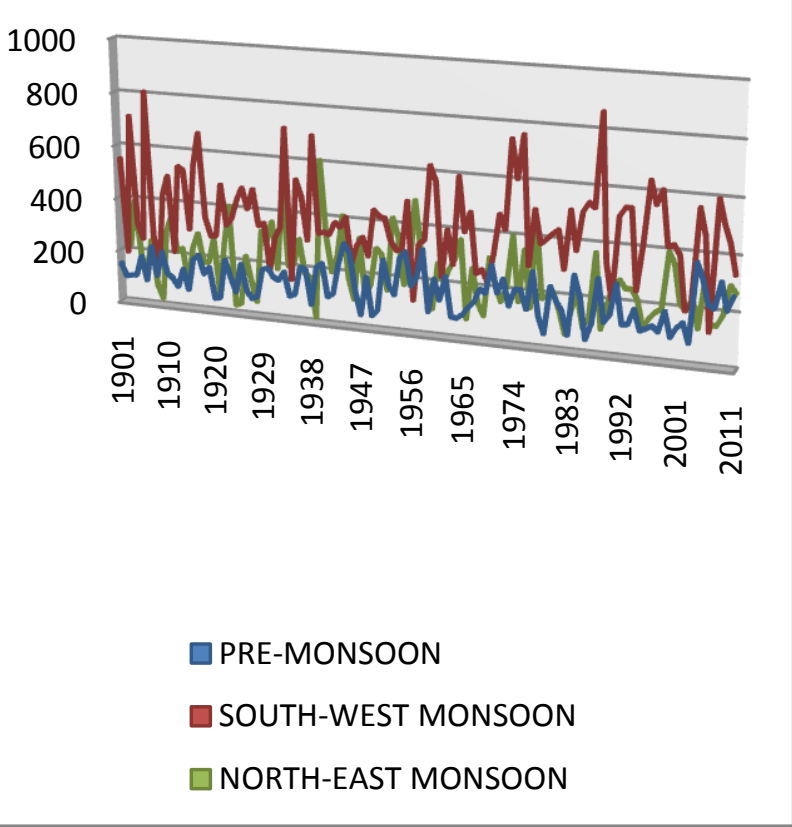

Fig 7 seasonal variation of rainfall(1901-2011) 


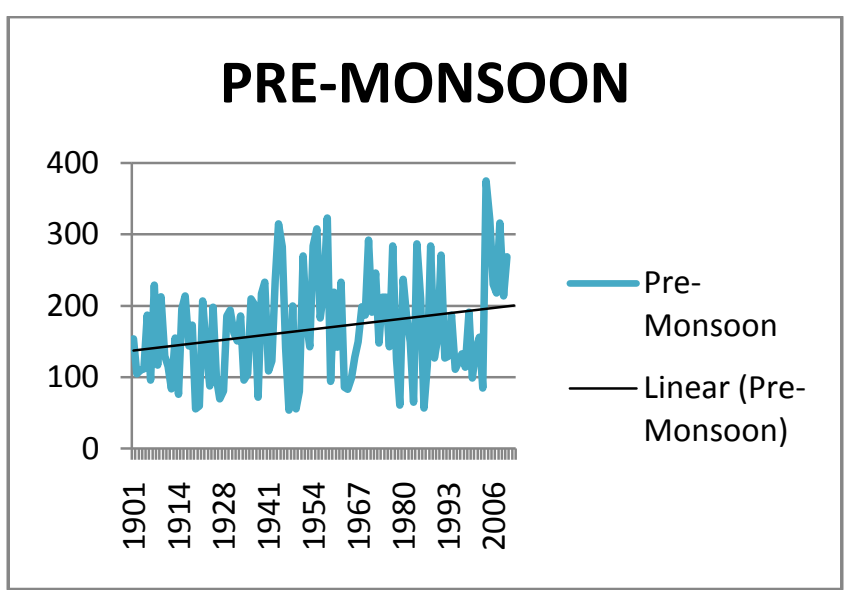

Fig 8 Pre monsoon rainfall (1901-2011)

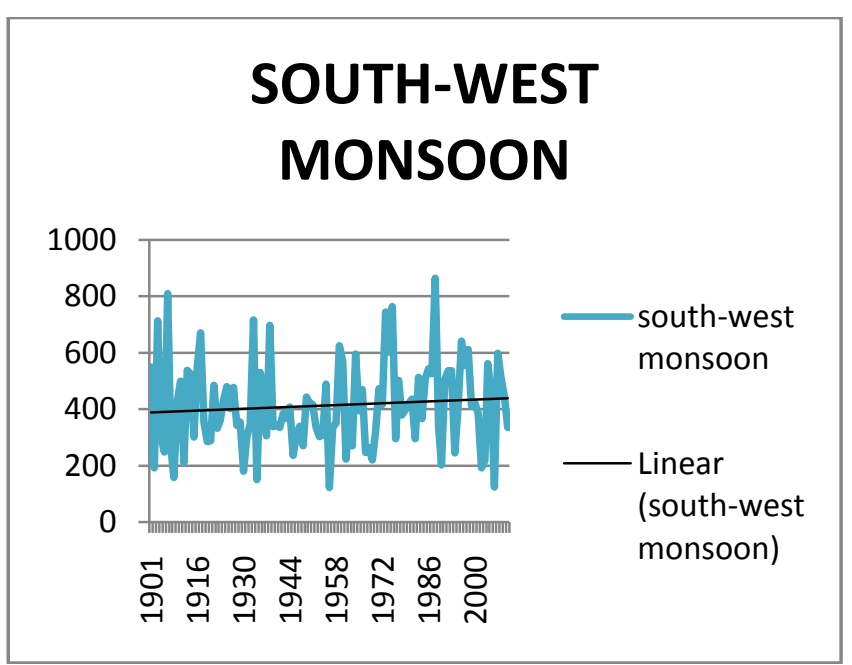

Fig 9 south west monsoon (1901-2011)

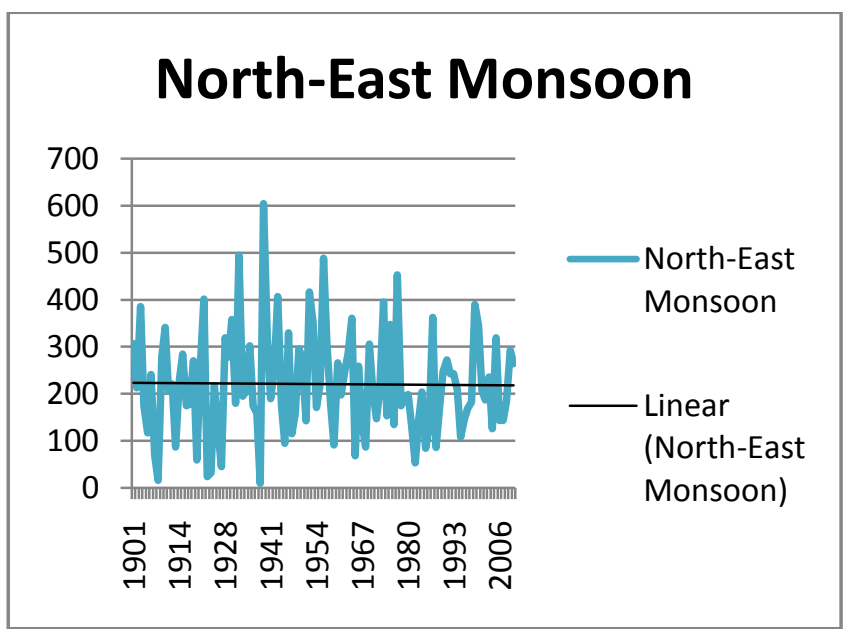

Fig 10 North east monsoon (1901-2011) 